\section{Revista 2020 \\ Med Vol. 28(2)}

julio-diciembre
-
ISSN: 0121-5256 · e-ISSN: 1909-7700
Editorial Neogranadina

\title{
Análisis de beta-glucosidasa en sangre seca recolectada en papel filtro (DBS), reporte de un nuevo método aplicado a población control y pacientes con sospecha de enfermedad de Gaucher*
}

\author{
Ana Camila Vásquez-Salazara ${ }^{\mathrm{a}}$ Alfredo Uribe-Ardila ${ }^{\mathrm{b}}$
}

\begin{abstract}
Resumen: la enfermedad de Gaucher (GD) es el trastorno de almacenamiento lisosomal que se caracteriza por la deficiencia en la actividad enzimática de la $\beta$-glucosidasa (BGLU), lo que produce la acumulación de glucosilceramida en las células. Su diagnóstico se orienta a la valoración de la enzima en los leucocitos afectados. Se han realizado estudios en DBS para la actividad de BGLU en el seguimiento de poblaciones de alto riesgo; sin embargo, presentan interferencias relacionadas a leucopenias severas o expresión aumentada de la isoforma neutra de la enzima BGLU, molécula no relacionada con GD. El objetivo de este estudio fue la estandarización de un método de tamizaje en DBS (punch: $5 \mathrm{~mm}$ ) con el uso de 4-metilumbeliferil- $\beta$-D-glucósido y conduritol- $\beta$-epóxido. Se analizaron muestras de DBS de 395 individuos con sospecha clínica (población de alto riesgo o AR), 151 controles y 16 pacientes afectados, usando la elución de un corte de 5 mm ( $\approx 10 \mu$ de sangre) en $300 \mu$ le Tritón X-100/(0,5\%). Como resultados, se obtuvieron los rangos, AR: 0,84-26,92 nmol/ml/h, controles: 3,56$8,92 \mathrm{nmol} / \mathrm{ml} / \mathrm{h}(\mathrm{M}=5,56, \mathrm{DS}=1,15)$ y pacientes confirmados con GD: 0,82- $2,88 \mathrm{nmol} / \mathrm{ml} / \mathrm{h}(\mathrm{M}=1,64$, DS $=0,57)$. El punto de corte entre deficientes y controles fue $3,22 \mathrm{nmol} / \mathrm{ml} / \mathrm{h}$, obtenido a partir de análisis RoC (99\% confianza, $100 \%$ sensibilidad y $100 \%$ especificidad). El protocolo permitió evidenciar la deficiencia en todos los casos de GD, confirmados mediante el análisis en paralelo de la enzima en aislamiento leucocitario. Se recomienda el uso del CBE y realizar la elución del corte a $5 \mathrm{~mm}$, a fin
\end{abstract}

* Artículo de investigación.

a Centro de Investigaciones en Bioquímica (CIBI), Departamento de Ciencias Biológicas, Universidad de los Andes. Bogotá, Colombia. Correo electrónico: ac.vasquez10@uniandes.edu.co ORCID: https://orcid. org/0000-0001-8788-116X

b Centro de Investigaciones en Bioquímica (CIBI), Departamento de Ciencias Biológicas, Universidad de los Andes. Bogotá, Colombia. Correo electrónico: jeuribe@uniandes.edu.co ORCID: https://orcid.org/00000001-5115-9597 
de llevar a cabo la valoración enzimática con un volumen mayor aproximado de sangre y en ausencia de la actividad generada por la isoforma neutra.

Palabras clave: enfermedad de Gaucher; $\beta$-glucosidasa; glucocerebrosidasa; sangre seca en papel filtro

Recibido: 04/12/2019. Aceptado: 30/06/2020.

Disponible en línea: 04/06/2021

Cómo citar: Vásquez-Salazar AC, Uribe-Ardila A. Análisis de beta-glucosidasa en sangre seca recolectada en papel filtro (DBS), reporte de un nuevo método aplicado a población control y pacientes con sospecha de enfermedad de Gaucher. Rev. Med. [Internet]. 4 de junio de 2021 [citado 4 de junio de 2021];28(2):35-8. Disponible en: https://revistas.unimilitar.edu.co/index.php/rmed/article/view/4857

\title{
Beta-Glucosidase analysis in collected dried blood spots (DBS), report of a new method applied to the control population and patients with suspicion of Gaucher disease
}

\begin{abstract}
GD) is a lysosomal storage disorder characterized by a deficiency in the enzymatic activity of $\beta$-glucosidase (BGLU), resulting in the accumulation of glucosylceramide in cells. Its diagnosis is aimed at checking the enzyme in the affected leukocytes. Studies have been conducted on dried blood spots (DBS) for BGLU activity to monitor high-risk populations; however, they exhibit interferences related to severe leukopenias or increased expression of the neutral BGLU isoform, a molecule not related to GD. This study intends to standardize a screening method on DBS (punch: 5 $\mathrm{mm}$ ) using 4-methylumbelliferyl- $\beta$-D-glucoside and conduritol- $\beta$-epoxide (C $\beta E$ ). DBS samples from 395 individuals clinically suspected of GD (high-risk or HR population), 151 controls, and 16 affected patients were analyzed using the elution of $5 \mathrm{~mm}$ punches $(\approx 10 \mu \mathrm{l}$ of blood) in $300 \mu \mathrm{l}$ of Triton X-100/ (0.5\%). As a result, the following ranges were obtained; HR: $0.84-26.92 \mathrm{nmol} / \mathrm{ml} / \mathrm{h}$, controls: $3.56-8.92$ $\mathrm{nmol} / \mathrm{ml} / \mathrm{h}(M=5.56, S D=1.15)$, and patients with confirmed GD: $0.82-2.88 \mathrm{nmol} / \mathrm{ml} / \mathrm{h}(M=1.64, S D=$ 0.57). The cut-off point between patients with GD and controls was $3.22 \mathrm{nmol} / \mathrm{ml} / \mathrm{h}$, obtained from ROC analysis (99\% CI, $100 \%$ sensitivity, and $100 \%$ specificity). The protocol revealed a deficiency in all GD cases, confirmed by parallel BGLU analysis in isolated leukocytes. The use of CBE and the elution of $5 \mathrm{~mm}$ punches are recommended for enzymatic evaluation with a higher approximate volume of blood and in the absence of neutral isoform activity.
\end{abstract}

Keywords: Gaucher disease; $\beta$-glucosidase; glucocerebrosidase; dried blood spots

\section{Análise de $\beta$-glucosidase em sangue seco coletado em papel filtro (DBS), relatório de um novo método aplicado à população controle e a pacientes com suspeita de doença de Gaucher}

Resumo: a doença de Gaucher (GD) é o trastorno de armazenamento lisosomal caracterizado pela deficiência na atividade enzimática da $\beta$-glucosidase (BGLU), o que produz a acumulação de glucossilceramida nas células. Seu diagnóstico está orientado à avaliação da enzima nos leucócitos afetados. Foram realizados estudos em DBS para a atividade de BGLU no seguimento de populações de alto risco; contudo, são apresentadas interferências relacionadas a leucopenias graves ou a expressão aumentada da isoforma neutra da enzima BGLU, molécula não relacionada com GD. O objetivo 
deste estudo foi a padronização de um método de tamisação emDBs (punch: 5 mm) com o uso de 4-metilumbeliferil- $\beta$-D- glicosídeo e conduritol- $\beta$-epóxido. Foram analisadas amostras de DBS de 395 indivíduos com suspeita clínica (população de alto risco ou AR), 151 controles e 16 pacientes afetados, usando a eluição de um corte de 5 mm ( $\approx 10 \mu \mathrm{l}$ de sangue) em $300 \mu \mathrm{l}$ de Tritão X-100/(0,5 \%). Como resultados, foram obtidos os intervalos: AR: 0,84-26,92 nmol/ml/h, controles: 3,56-8,92 nmol/ml/h $(\mathrm{M}=5,56$, DS $=1,15)$ e pacientes confirmados com GD: 0,82- 2,88 nmol/ml/h $(\mathrm{M}=1,64, \mathrm{DS}=0,57) .0$ ponto de corte entre deficientes e controles foi 3,22 nmol/ml/h, obtido a partir de análise Roc (99\% confiança, $100 \%$ sensibilidade e 100 \% especificidade). O protocolo permitiu evidenciar a deficiência em todos os casos de GD, confirmados mediante a análise em paralelo da enzima em isolamento leucocitário. É recomendado o uso do CBE e a realização da eluição do corte a $5 \mathrm{~mm}$, a fim de implementar a avaliação enzimática com um volume maior aproximado de sangue e em ausência da atividade gerada pela isoforma neutra.

Palavras-chave: doença de Gaucher; $\beta$-glucosidase; glucocerebrosidase; sangue seco em papel filtro 


\section{Introducción}

Las enfermedades raras (ER) son condiciones causadas en su mayoría por defectos genéticos, en las cuales se afecta un órgano o varios tejidos en el organismo por lo que pueden llegar a considerarse como multisistémicas (1). Este tipo de patologías se clasifican dentro de las afecciones de muy baja prevalencia, ya que la padecen menos de 200000 personas en una población (2). En esta categoría de ER, las enfermedades metabólicas son condiciones hereditarias en las cuales se bloquea una vía metabólica a causa de una mutación específica. Las enfermedades de depósito lisosomal, por su parte, son alteraciones que se producen por mutaciones en enzimas, cuya función es degradar macromoléculas en partículas más pequeñas, de manera que el organismo las puede digerir más fácilmente (3). Como consecuencia, se acumula el sustrato correspondiente en células y tejidos, lo que afecta de forma grave el correcto funcionamiento del organismo.

Dependiendo del sustrato acumulado, las enfermedades de depósito lisosomal pueden clasificarse en: esfingolipidosis (enfermedad de Fabry, enfermedad de Gaucher), mucopolisacaridosis, oligosacaridosis (fucosidosis, manosidosis), glucogenosis como la enfermedad de Pompe y mucolipidosis (2).

La enfermedad de Gaucher es una condición metabólica que cuenta con una prevalencia del $41,7 \%$ en las enfermedades de depósito lisosomal en Colombia (4), lo que la posiciona como la esfingolipidosis más común en el país. Consta de una mutación en el gen de la glucocerebrosidasa (GBA) localizado en el brazo largo del cromosoma 1 (1q21.1) (5), por lo que codifica para una proteína cuya actividad catalítica es deficiente. De este modo, se bloquea la degradación de la glucosilceramida en ceramida y glucosa $(6,7)$, lo que provoca que se acumule la sustancia lipídica en los lisosomas de las células pertenecientes al Sistema Fagocítico Mononuclear (SFM): leucocitos de sangre circulante y macrófagos que van a diferentes tejidos (8). Estas células cargadas de glucocerebrósido se denominan Células de Gaucher (CG), se localizan principalmente en bazo, hígado y médula ósea, y causan patologías como hepatoesplenomegalia, pancitopenia y complicaciones óseas $(2,9)$, lo que conduce al desarrollo de una enfermedad multisistémica en el paciente. Aunque se han descrito más de 300 mutaciones para el gen GBA, a finales de 1980 se identificaron los alelos N370S y L444P como mayormente asociados con las variantes de la enfermedad (2,6,10-12). Cabe destacar que esta alteración se hereda de forma autosómica recesiva, es decir, se necesitan dos copias del gen anormal (uno dado por el padre y otro dado por la madre) para que el individuo desarrolle la enfermedad (13).

A partir de lo anteriormente mencionado, la enfermedad de Gaucher constituye una variedad de presentaciones fenotípicas que se pueden clasificar en tres tipos: Tipo I o no neuropático, hace parte de la variante más común e incluye afectación hematológica (leucopenia severa, anemia, trombocitopenia), afectación visceral (hepatoesplenomegalia), afectación ósea (osteopenia, deformidades óseas) y en algunos casos, aunque con menor frecuencia, está asociada con manifestaciones pulmonares y cardiacas y afectación renal (2). El Tipo II o neuropático agudo es la variante más grave, ya que incluye parálisis ocular, hiperesplenismo, dificultades en la alimentación y alteraciones neurológicas con deterioro cerebral progresivo, lo que puede llevar al recién nacido a la muerte en un período de entre 3 meses y 2 años $(14,15)$. Finalmente, el Tipo III o neuropático crónico incluye tanto la afectación visceral como la afectación neurológica, aunque precoz y más leve $(8,9)$.

Con el fin de tratar esta variedad de cuadros clínicos, existe la Terapia de Reemplazo Enzimático (TRE), que consta de administrar una enzima recombinante (imiglucerasa) vía intravenosa, de manera que se reducen las alteraciones y los síntomas, a la vez que se mejora la calidad de vida del paciente $(2,13,16)$. Adicionalmente, en los años 2010 y 2012 se desarrollaron dos fármacos efectivos para el tipo I de la enfermedad de Gaucher como parte de la Terapia de Reemplazo Enzimático: Velaglucerasa alfa (vPriv) en 2010 y Taliglucerasa alfa en 2012. El Velaglucerasa alfa se produjo a partir de líneas celulares de fibroblastos 
humanos (17), mientras que la Taliglucerasa alfa fue producida con células de zanahoria genéticamente modificadas (17). En este orden de ideas, es importante mencionar la Terapia de Reducción de Sustrato (TRS), una técnica que busca reducir la carga de glucosilceramida en el lisosoma $(17,18)$. En 2002 se desarrolló Miglustat, un medicamento que logra atravesar la barrera hematoencefálica, por lo cual se concibió para el manejo de los tipos II y III de la enfermedad de Gaucher. No obstante, causa la mayor incidencia de reacciones adversas, por lo que está aprobado solo para pacientes que no pueden tomar la TRE (17). En la actualidad se encuentra otro candidato para la TRS, cuyo nombre es Eliglustat y se encuentra en la fase 3 de ensayos clínicos; sin embargo, no penetra la barrera hematoencefálica (17).

Como parte de los esfuerzos por dilucidar los aspectos relacionados con esta enfermedad, distintos investigadores han reportado la presencia de dos isoformas para la $\beta$-glucosidasa en leucocitos, con diferencias en cuanto a su termoestabilidad en los individuos afectados e incluso con un comportamiento diferencial en los individuos control $(6,19)$. Aunque ambas isoenzimas pueden degradar el sustrato que se les proporciona en el laboratorio, solo una de ellas está relacionada con la degradación del sustrato natural, de modo que es la única que se involucra directamente con el desarrollo de la enfermedad de Gaucher $(6,20,21)$. Por este motivo, los autores han sometido las isoenzimas a diferentes reactivos, a fin de estimular la $\beta$-glucosidasa de interés clínico e inhibir la isoforma neutra, para obtener actividades enzimáticas cada vez más cercanas a lo que ocurre naturalmente en el lisosoma. Cabe destacar la importancia de identificar y eliminar la interferencia que causa la isoforma neutra (aquella que no está relacionada con GD, pero sí degrada el sustrato artificial), puesto que se encuentra en una proporción del $15 \%$ en población control y hasta del $100 \%$ en pacientes afectados por Gaucher (22).

Dada la complejidad de la enfermedad, su diagnóstico se lleva a cabo mediante la determinación de la $\beta$-glucosidasa en leucocitos de sangre periférica, a través de un método fluorométrico que se considera altamente reproducible $(7,21)$ y se denomina como gold standard. En Colombia este procedimiento se realiza en centros especializados que se encuentran solo en algunas de las principales ciudades, lo que significa un limitante para el estudio de un gran número de afectados que no cuentan con acceso directo a estos centros, puesto que se hallan en territorios alejados del país. Además, la técnica genera costos adicionales debido a que las muestras leucocitarias deben enviarse desde las distintas ciudades por medio de una estricta cadena de frío, de modo que se asegure su calidad y correcto mantenimiento. Por consiguiente, las limitaciones geográficas con las que cuenta el país derivan en un número subestimado de pacientes diagnosticados y diagnósticos tardíos que incluso pueden llevar a la muerte de los pacientes (4).

Por lo anterior, algunos autores plantean la posibilidad de llevar a cabo una prueba de tamizaje como herramienta de aproximación diagnóstica, que se fundamenta en el uso de sangre total representada en una gota de sangre seca sobre papel filtro (DBS = Dried Blood Spot), en la que se puede determinar la actividad de la $\beta$-glucosidasa (14). Esta técnica facilita el análisis de muestras a gran escala con un costo razonable, debido a que no se requieren condiciones estrictas de frío para su transporte y este puede llevarse a cabo por medio de servicios de mensajería nacional. Así mismo, se denota la importancia de la prueba de tamizaje para facilitar la identificación temprana de los pacientes (14), en especial, aquellos con enfermedad de Gaucher Tipo II que en su mayoría corresponden a la población pediátrica, puesto que el manejo oportuno de la enfermedad lleva a una mejora en el desarrollo motor, así como en la afectación visceral y los parámetros de crecimiento (15). Sin embargo, el tamizaje cuenta con algunas interferencias que no han hecho posible su implementación: la expresión aumentada de la isoforma neutra que produce falsos negativos $(14,22,23)$ y la alta tasa de falsos positivos que se ocasiona por la clasificación de individuos leucopénicos como enfermos de Gaucher (24). A lo anterior, se suma la baja correlación que presenta el tamizaje con la prueba diagnóstica (25). Por esto, algunos autores hacen hincapié en la importancia de realizar estudios de comportamiento enzimático dada la heterogeneidad de la 
enfermedad (6) y a la relevancia de desarrollar la prueba de tamizaje para detectar la real incidencia de la enfermedad en países donde el muestreo regular es limitado (26).

El presente estudio tiene como propósito estandarizar y correlacionar un método de tamizaje en DBs para la enfermedad de Gaucher, con un corte de $5 \mathrm{~mm}$ y con el uso del sustrato fluorogénico 4-metilumbeliferil- $\beta$-D-glucósido y el inhibidor para la $\beta$-glucosidasa (conduritol- $\beta$-epóxido). Lo anterior, a fin de establecer los valores de referencia para la población colombiana mediante la evaluación de individuos controles y población de alto riesgo, para determinar la sensibilidad y especificidad del método en cuanto a la reducción de falsos positivos y falsos negativos. La técnica aquí reportada, constituye una herramienta clave para la orientación clínica de los pacientes y un medio para alcanzar oportunamente su diagnóstico, lo que se traduce en una intervención temprana que cambie el curso de la enfermedad y mejore la calidad de vida de los pacientes y sus familias.

En este estudio se incluye un nuevo método, el cual consiste en eluir el corte $(5 \mathrm{~mm})$ en Tritón $\mathrm{X}-100 \mathrm{y}$ agua ultra pura, con el fin de obtener un mayor volumen aproximado de sangre. Así mismo, incorpora reactivos para estimular e inhibir la $\beta$-glucosidasa y su isoforma neutra, con el propósito de lograr resultados más acertados de la actividad enzimática relacionada con la patología.

\section{Materiales y métodos}

\section{Recolección de muestras}

Se colectaron 395 muestras de papel filtro correspondientes a pacientes con edades entre 1,2 meses y 86,1 años que contaban con manifestaciones clínicas propias de una anomalía en el metabolismo de esfingolípidos, por lo que se tenía sospecha de que podrían estar afectados por la enfermedad de Gaucher. Como controles positivos se recolectaron 16 muestras de pacientes con edades entre 1,25 y 76,1 años que fueron diagnosticados con GD a través de la técnica gold standard y no habían recibido la enzima recombinante al momento de llevar a cabo la extracción de sangre. A fin de establecer los valores de referencia en la población y como controles normales de la prueba, se colectaron 151 muestras pertenecientes a individuos con edades entre 1,2 meses y 78,56 años.

En el estudio se evaluaron 41 muestras enviadas desde Perú, que pertenecían a pacientes entre los 1,2 meses y los 67,6 años con sospecha de enfermedad de depósito lisosomal. Adicionalmente, en el grupo de afectados en el estudio, se analizó un paciente peruano con la edad de 6,7 años. Estas muestras recibieron el mismo procedimiento experimental y analítico que las muestras de población colombiana.

\section{Preparación de DBS}

Las muestras fueron tomadas vía intravenosa y se colectaron por goteo directo en tarjetas de papel filtro Whatman 903 provistas por GE Healthcare (Estados Unidos), conocidas por ser tarjetas de recolección protectoras de proteína con fines de investigación. Posteriormente, las muestras se dejaron secar a temperatura ambiente por un período de entre 8 y 12 horas, y se llevaron a $4{ }^{\circ} \mathrm{C}$ de refrigeración en bolsas de cierre hermético para prevenir su deterioro. En nuestro laboratorio se prepararon las muestras correspondientes a los individuos control, las demás fueron enviadas directamente a nuestro laboratorio que sirve como un punto de referencia para el diagnóstico y seguimiento de los pacientes con la enfermedad de Gaucher.

\section{Preparación del eluido}

Los ensayos se llevaron a cabo con la elución del corte de $5 \mathrm{~mm}$ en una solución a temperatura ambiente de $150 \mu \mathrm{l}$ de Tritón X-100 0,5 \% (v/v) y $150 \mu \mathrm{l}$ de agua ultrapura en tubos Eppendorf de 1,5 ml. Luego las muestras se llevaron a agitación orbital sobre una superficie plana en un agitador vibratorio de plataforma vibrante Titramax 1000 de Heidolph (Schwabach, Alemania) durante una hora a $900 \mathrm{rpm}$ temperatura ambiente. Así mismo, se centrifugaron por 5 minutos. Finalmente, se retiró el papel filtro y se agregaron las muestras a cada pozo en volúmenes de $20 \mu \mathrm{l}$ por triplicado para cada individuo. Para este fin, se usaron microplacas de polipropileno de 96 pozos de color negro 
para minimizar el ruido de fondo en la medición de la fluorescencia. Las placas se cubrieron con láminas de aluminio para sellado térmico, provistas por Corning (Lowell, EE. UU.), con el propósito de evitar la evaporación de las muestras, y se llevaron a agitación durante 5 minutos para lograr una mezcla homogénea de los reactivos.

\section{Ensayo de actividad enzimática}

El procedimiento experimental se realizó teniendo en cuenta los estudios previos de $(20,27)$ con relación al efecto del taurodeoxicolato de sodio (TDC) y el conduritol- $\beta$-epóxido (CBE) sobre la $\beta$-glucosidasa. La metodología se basó en el protocolo propuesto por $(25,26)$, aunque se modificó el corte a $5 \mathrm{~mm}$ de diámetro $(\approx 10 \mu \mathrm{l}$ de sangre) y los volúmenes de buffers y reactivos así: $20 \mu \mathrm{l}$ de buffer citrato fosfato $0,4 \mathrm{M}, \mathrm{pH} 5.2$ con TDC $0,5 \%$ (\% p/v) y CBE 0,5 mM. Los análisis sobre una gota de sangre seca sobre papel filtro (DBS) se llevaron a cabo empleando el sustrato artificial 4-metilumbeliferil- $\beta$-D-glucósido con una concentración de $20 \mathrm{mM}$ en el buffer de reacción. Este sustrato está marcado con 4-metil-umbeliferona (4-MU) provisto por Sigma (EE. UU.). El volumen de sangre aproximado en los $5 \mathrm{~mm}$ de corte, se calculó a partir del volumen estandarizado en $1,2 \mathrm{~mm}(\approx 0,52 \mu \mathrm{l}$ de sangre) reportado previamente (4,28-30).

Para evaluar la degradación inespecífica del sustrato en el buffer de reacción y la interferencia causada por la isoforma y la hemoglobina, se incluyó un volumen adicional igual al que fue usado en las muestras y se agregó el inhibidor (CBE) de la $\beta$-glucosidasa. Estos pozos corresponden a los blancos del procedimiento experimental. La diferencia entre los valores de fluorescencia de las muestras y los blancos permitió diferenciar la actividad enzimática real de la degradación inespecífica.

El tiempo de incubación fue de 24 horas a $37^{\circ} \mathrm{C}$ por agitación orbital a $120 \mathrm{rpm}$ en un Unimax 1010 (incubador/agitador) de Heidolph (Schwabach, Alemania) y la reacción se detuvo agregando el buffer de parada ( $150 \mu \mathrm{l}$ de glicina carbonato 0,17 $\mathrm{M}, \mathrm{pH}$ 10.5) a las muestras y blancos. Como lector de fluorescencia, se usó el equipo SpectraMax ${ }^{\circledR}$ M2 provisto por Molecular Devices (excitación 360 nm, emisión $450 \mathrm{~nm}$ ) y los resultados se compararon con una curva de calibración de 10 puntos de 4-metil-umbeliferona (4-MU). La actividad enzimática fue expresada como nanomoles de sustrato hidrolizado por mililitro de sangre por hora (nmol/ml/h).

\section{Porcentaje de recuperación enzimática}

Con el fin de determinar el porcentaje que se recuperó de la enzima de interés en el eluido, se tomaron muestras de pacientes y controles al azar en cada montaje, y se evaluaron para hexosaminidasa como enzima control. Cabe destacar que las muestras se tomaron del mismo eluido del que se determinó la actividad de $\beta$-glucosidasa, y se agregaron de igual manera en cada pozo en volúmenes de $20 \mu \mathrm{l}$ por duplicado para cada individuo. El volumen del buffer y reactivos se tomaron en cuenta según lo reportado previamente por (4), así: $60 \mu \mathrm{l}$ de buffer citrato fosfato $0,01 \mathrm{M}, \mathrm{pH} 4.4$ con el sustrato artificial 4-metilumbeliferil-2-acetamido-2-deoxy- $\beta$-D-glucopiranósido $3 \mathrm{mM}$ correspondiente a la concentración en papel de filtro. La incubación de las muestras se llevó a cabo por 1 hora a $37{ }^{\circ} \mathrm{C}$ por agitación orbital a $120 \mathrm{rpm}$ en un Unimax 1010 (incubador/agitador) de Heidolph (Schwabach, Alemania). La reacción se detuvo agregando $150 \mu \mathrm{l}$ del buffer de parada glicina carbonato $0,17 \mathrm{M}, \mathrm{pH} 10$ a las muestras y los blancos. Un volumen de sustrato igual al que se usó para las muestras $(60 \mu \mathrm{l})$, se incubó en un tubo Eppendorf separado y se agregó en los pozos de los blancos para evaluar la degradación inespecífica en el buffer de reacción. Del mismo modo, la lectura se llevó a cabo por medio del equipo SpectraMax ${ }^{\oplus}$ 2 (excitación $360 \mathrm{~nm}$, emisión $450 \mathrm{~nm}$ ) y los resultados se compararon con una curva de calibración de 4-MU. El porcentaje de recuperación se calculó teniendo en cuenta la actividad enzimática obtenida con el eluido y la actividad enzimática obtenida con el protocolo de DBS [1,2 mm de corte y un volumen de $\approx 0,52 \mu$ l de sangre, reportado por (4)].

Todas las muestras fueron procesadas por duplicado (en el caso de hexosaminidasa) y triplicado (en el de $\beta$-glucosidasa) para establecer el 
coeficiente de variación intraensayo. En este orden de ideas, se evaluó la variación interensayo por medio de las muestras de los controles normales que se evaluaron en diferentes placas y en distintas fechas. Cabe mencionar que en cada placa se incluyeron muestras de pacientes, controles normales y pacientes afectados para evaluar la viabilidad del ensayo.

De forma paralela a la valoración enzimática en el eluido, se llevó a cabo la confirmación de todas las muestras en aislamiento de leucocitos siguiendo el protocolo propuesto por (31). La cantidad de la proteína en las muestras se determinó por los métodos de Lowry у вса. A partir de estos valores, se estableció la correlación entre el nuevo método (eluido) y la técnica confirmatoria (gold standard).

\section{Cinética enzimática}

Se realizó una cinética enzimática que incluyó tres controles normales y un paciente afectado, con ocho tiempos de incubación $(1,2,4,8,10,21,24$ y 34 horas). Cabe destacar que el único parámetro que se cambió correspondió al tiempo de incubación de la $\beta$-glucosidasa.

\section{Aspectos éticos}

Todas las muestras que se obtuvieron para el análisis y almacenamiento contaron con un consentimiento informado que tuvo la firma de los pacientes y controles. El consentimiento incluyó un formulario aprobado por el Comité de Ética del Departamento de Ciencias Biológicas de la Universidad de los Andes. El estudio se realizó en correspondencia con la Declaración de Helsinki.

\section{Análisis estadístico}

Se realizó con el software IBM SPSS Statistic 19 de SPSS Inc. (Chicago, EE. UU.), que generó la curva ROC por medio de la cual se determinó el punto de corte teniendo en cuenta el límite de las actividades enzimáticas entre los controles y pacientes afectados. Así mismo, se estableció un coeficiente de variación (Cv) inter e intraensayo como un valor promedio de las muestras en la misma placa y en placas con diferentes fechas a manera de repeticiones. Para evaluar la distribución de los datos se realizó la prueba de Shapiro-Wilk. Posteriormente, se hizo un Kruskal-Wallis test acompañado del Tukey-Kramer test (no paramétrico) para determinar si existían diferencias significativas entre las actividades enzimáticas de los grupos de individuos en el estudio.

\section{Resultados y discusión}

Los valores de referencia para la $\beta$-glucosidasa en la población colombiana se establecieron a partir del análisis enzimático de las 151 muestras correspondientes a la población control. Así mismo, se evaluaron 395 pacientes que contaban con presentaciones clínicas propias de enfermedad lisosomal y 16 pacientes afectados confirmados con la prueba diagnóstica, con un total de 562 muestras procesadas en DBS y en gold standard. En este sentido, se obtuvo un rango de 0,82 a $2,88 \mathrm{nmol} /$ $\mathrm{ml} / \mathrm{h}$ y de 3,56 a $8,92 \mathrm{nmol} / \mathrm{ml} / \mathrm{h}$ para afectados y controles, respectivamente. Además, calculamos como punto de corte 3,22 $\mathrm{nmol} / \mathrm{ml} / \mathrm{h}$ (análisis ROC-IBM SPSS Statistics) entre controles normales e individuos afectados. La curva Roc obtenida se muestra en la Figura 1. A. (confianza $=99 \%$, sensibilidad $=100 \%$, especificidad $=100 \%$ ). También se determinó 24 horas como el tiempo más adecuado para llevar a cabo el protocolo, dado que en este tiempo se obtuvo una separación promedio considerable entre las actividades de los controles y el individuo afectado (Figura 1. B.), además que otros autores han reportado ensayos con BGLU en tiempos de incubación prolongados (6). Los resultados de las actividades enzimáticas se detallan en la Tabla 1, así como las condiciones de incubación se pueden observar en la Tabla 2. 
Tabla 1. Resultados de las actividades enzimáticas en DBS para controles ${ }^{a}$ y afectados

\begin{tabular}{|c|c|c|c|c|c|c|}
\hline $\begin{array}{c}\text { Enzima: } \\
\beta \text {-glucosidasa }\end{array}$ & $\mathbf{n}$ & $\begin{array}{l}\text { Actividad (nmol/ml/h) } \\
\text { rango }\end{array}$ & $\mathrm{CV}_{1}$ & $\mathrm{CV}_{2}$ & Valor-p $p^{b}$ & $\begin{array}{l}\text { Punto de } \\
\text { corte }^{c}\end{array}$ \\
\hline $\begin{array}{c}\text { Afectados } \\
\text { (rango de edad: 1,25-76,1 años) }\end{array}$ & 16 & $\begin{array}{c}0,82-2,88 \\
M: 1,64 . \text { DS: } 0,57\end{array}$ & 4,40 & 4,9 & $<0,0001$ & 3,22 \\
\hline $\begin{array}{l}\text { Controles } \\
\text { (rango de edad: 1,2 meses-78,56 años) }\end{array}$ & 151 & $\begin{array}{c}3,56-8,92 \\
M: 5,56 . \mathrm{DS}: 1,15\end{array}$ & & & & \\
\hline
\end{tabular}

Fuente: elaboración propia.

a De acuerdo con la prueba de Shapiro-Wilk, el grupo control no mostró una distribución normal ( $p<0,05$ con un porcentaje del $95 \%$ de confianza).

b El valor $p$ detallado corresponde al resultado de la prueba Tukey-Kramer para comparar las actividades enzimáticas entre los individuos afectados y los controles sanos.

c Se obtuvo a partir del análisis Roc (Receiver Operating Characteristics) teniendo en cuenta las actividades enzimáticas entre afectados y controles ( $99 \%$ de confianza, $100 \%$ de especificidad, $100 \%$ sensibilidad y área bajo la curva de 1). Los parámetros de sensibilidad, especificidad y área bajo la curva corresponden a la capacidad discriminatoria de la prueba para clasificar a sanos y enfermos dentro de su grupo respectivo de manera correcta.

$\mathrm{CV}_{1}$ : coeficiente de variación intraensayo (\%).

$\mathrm{CV}_{2}$ : Coeficiente de variación interensayo (\%)

Tabla 2. Condiciones de incubación para las enzimas lisosomales en el estudio

\begin{tabular}{|c|c|c|c|c|}
\hline Enzima & Buffer de reacción & $\begin{array}{c}\text { Sustrato } \\
\text { (concentración en el } \\
\text { buffer de reacción) }\end{array}$ & $\begin{array}{c}\text { Tiempo } \\
\text { incubación/ } \\
\text { temperatura }\end{array}$ & Buffer de parada \\
\hline$\beta$-glucosidasa ${ }^{a}$ & $\begin{array}{c}20 \mu \mathrm{l} \text { de buffer citrato fosfato } 0,4 \mathrm{M}, \mathrm{pH} \\
5.2 \text { con taurodeoxicolato de sodio al } \\
0,5 \% \text {. Conduritol- } \beta \text {-epóxido } 0,5 \mathrm{mM}^{\mathrm{b}} \\
\text { únicamente para los blancos. }\end{array}$ & $\begin{array}{l}\text { 4-metilumbeliferil- } \beta \text { - } \\
\text { D-glucósido } 20 \text { mM }\end{array}$ & $24 \mathrm{~h} / 37^{\circ} \mathrm{C}$ & $\begin{array}{c}150 \mu \text { l de glicina } \\
\text { carbonato } 0,17 \mathrm{M} \text {, } \\
\text { pH } 10.5\end{array}$ \\
\hline Hexosaminidasa totala & $\begin{array}{l}60 \mu \mathrm{l} \text { de buffer citrato fosfato } 0,01 \mathrm{M} \text {, } \\
\qquad \mathrm{pH} 4.4\end{array}$ & $\begin{array}{l}\text { 4-metilumbeliferil- } \\
\text { 2-acetamido- } \\
\text { 2-deoxy- } \beta \text {-D- } \\
\text { glucopiranósido } 3 \mathrm{mM}\end{array}$ & $1 \mathrm{~h} / 37^{\circ} \mathrm{C}$ & $\begin{array}{c}150 \mu \mathrm{l} \text { de glicina } \\
\text { carbonato } 0,17 \mathrm{M} \text {, } \\
\text { pH } 10.5\end{array}$ \\
\hline
\end{tabular}

Fuente: elaboración propia.

a Para cada mezcla de reacción, el ensayo requirió la elución del corte de $5 \mathrm{~mm}$ de papel filtro ( $\approx 10 \mu \mathrm{l}$ de sangre) en una solución de $300 \mu$ l de Tritón X-100 al 0,5 \% y agua ultra pura. De esta muestra en elución se extraen $20 \mu$ l para la valoración enzimática.

b La evaluación de la $\beta$-glucosidasa en presencia de su inhibidor conduritol- $\beta$-epóxido (CBE) permite discriminar y eliminar la actividad de la isoforma neutra, que puede degradar el sustrato artificial, pero no está relacionada con la enfermedad de Gaucher (32).

La metodología no permitió una diferencia- ( $\mathrm{p}>0,05$ Tukey-Kramer test) (Figura 2). Lo anción significativa en las actividades enzimáticas terior, debido a que en el grupo de población de entre el grupo control y la población de alto riesgo alto riesgo se encontraron individuos con valores 
normales de BGLU, es decir, que no estaban afectados por la enfermedad de Gaucher. De la misma manera, se hallaron bajas expresiones de BGLU en individuos de este mismo grupo, pero que al realizar la confirmación con leucocitos, se encontró que no estaban afectados por Gaucher. Lo anterior puede tratarse de pacientes que cuentan con bajo recuento leucocitario como consecuencia de otras patologías con compromiso hematológico y hacen parte de los falsos positivos que pueden presentarse en el protocolo. En relación con esto, se ha reportado que una muestra con un número total de leucocitos bajo (paciente leucopénico) es uno de los factores críticos que afecta la actividad enzimática en la técnica de DBs (24) así como errores asociados con la preparación de la muestra y sus condiciones de almacenamiento $(4,14,24)$.

No obstante, esta metodología sí permitió determinar una diferencia significativa entre las actividades enzimáticas del grupo control y los individuos afectados por la enfermedad de Gaucher $(\mathrm{p}<0,001$ Tukey-Kramer test) (Tabla 1).

\section{A}

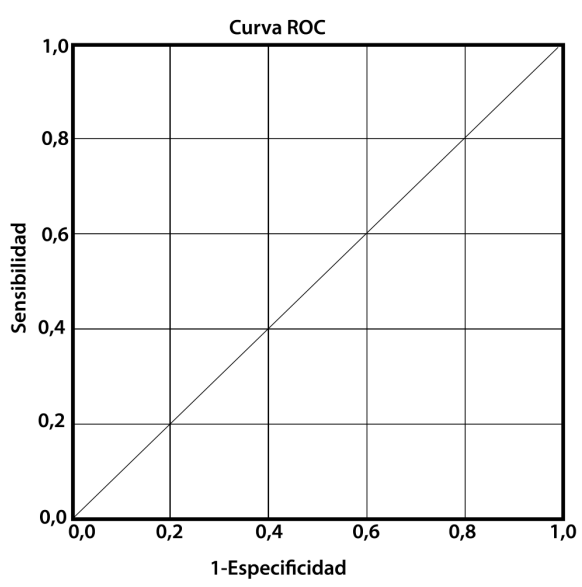

\section{B}

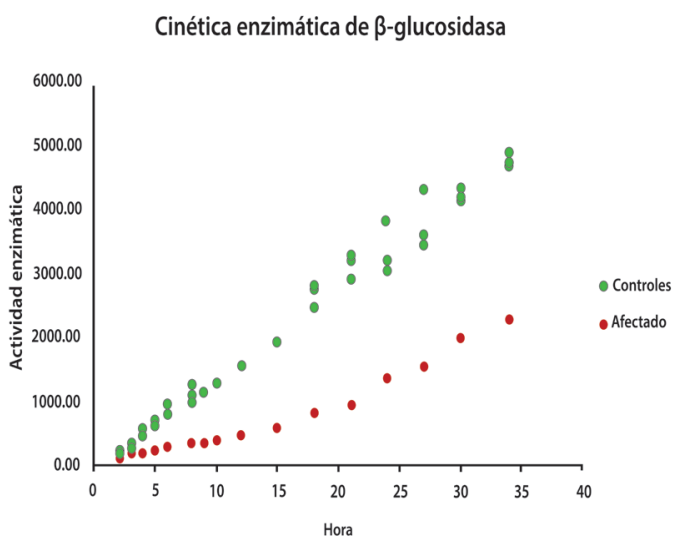

Figura 1. A. Curva Roc entre individuos afectados y controles normales (confianza $=99 \%$, sensibilidad $=100 \%$, especificidad $=100 \%$, AUC = 1). Se obtuvo como punto de corte 3,22 nmol/ml/h. B. Cinética enzimática de $\beta$-glucosidasa. Se determinó 24 h como el tiempo más adecuado para llevar a cabo el ensayo.

Fuente: elaboración propia.

Todas las muestras se evaluaron por triplicado y se eligieron controles internos de calidad con el fin de determinar la variabilidad del protocolo. A partir de esto, se obtuvo un coeficiente de variación intraensayo promedio de $4,4 \%$ y un coeficiente de variación interensayo de $4,9 \%(n=6)$, lo que demuestra que se trata de una técnica altamente reproducible.

El nuevo método que reportamos en el presente estudio trajo consigo los siguientes beneficios: en primer lugar, el líquido de elución es una solución que permite la extracción de la sangre del papel de filtro $(\approx 10 \mu \mathrm{l})$ sin que se vea mayormente comprometida la recuperación enzimática de la muestra. Para comprobar esto, se realizó el mismo procedimiento experimental para evaluar la $\beta$-glucosidasa con la enzima control hexosaminidasa en algunas muestras tomadas al azar por cada montaje, y se obtuvo un mínimo de recuperación enzimática del $70,19 \%$ en la muestra eluida $(n=$ 301 , rango entre 77,3 y $895,5 \mathrm{nmol} / \mathrm{ml} / \mathrm{h}, \mathrm{M}=298$, DS $=115,9)$. Los resultados para hexosaminidasa en DBS (eluido) se contrastaron con los resultados obtenidos en la técnica estandarizada para esta enzima en DBs (corte $=1,2 \mathrm{~mm}$ ) para cada uno de los individuos evaluados (datos no mostrados). 
Además, el eluido permite hacer réplicas u otras pruebas enzimáticas con el uso del mismo sobrenadante (26) lo que facilita el estudio de otras alteraciones metabólicas usando la misma muestra.

En segundo lugar, el corte de $5 \mathrm{~mm}$ mejora la reproducibilidad del protocolo al disminuir el error experimental asociado a este, ya que cuenta con un volumen mayor aproximado de sangre $(\approx 10 \mu \mathrm{l})$ en comparación con el volumen estandarizado para $1,2 \mathrm{~mm}(\approx 0,52 \mu \mathrm{l})$ que hace parte de la técnica DBs convencional para otras enzimas en Colombia (4). Lo anterior se encuentra estrechamente relacionado con la baja proporción de falsos positivos que se obtuvo en este estudio, que fue de aproximadamente el 12,6\%. No obstante, este porcentaje puede deberse a un efecto en el proceso de la estandarización y en mayor proporción a pacientes leucopénicos sin orientación clínica adecuada, por lo que estimamos que el 10\% sería el porcentaje máximo de falsos positivos asociado al protocolo.
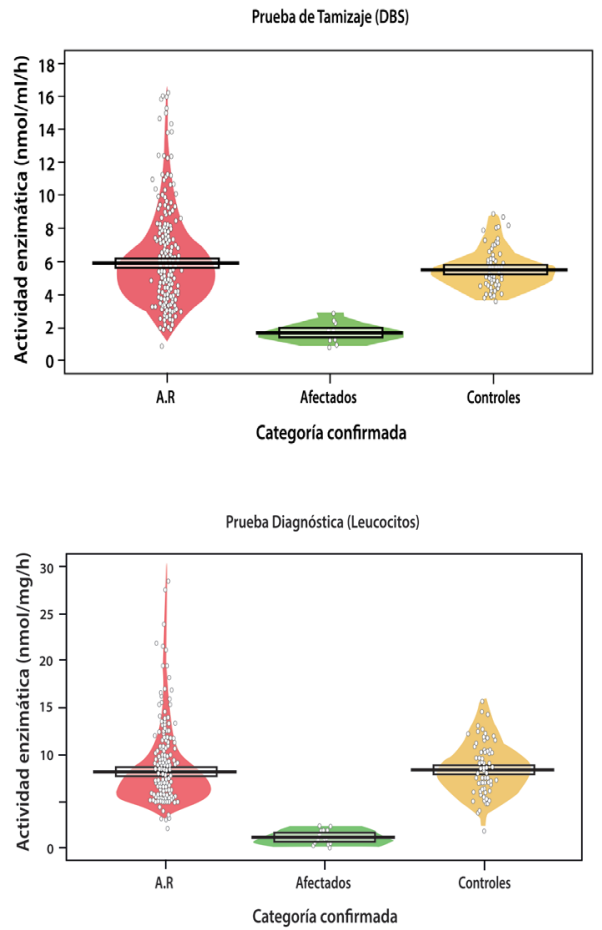

Figura 2. Comparación entre el tamizaje (DBS) y la prueba diagnóstica (leucocitos) para los grupos de individuos. Los violin plots demuestran que no hay sobrelapamiento entre los afectados con Gaucher y los individuos control $(n=464)$

Fuente: elaboración propia.
* La categoría "AR" alude al amplio grupo de individuos que hacen parte de la población de Alto Riesgo para la enfermedad de Gaucher. La categoría de "Afectados" hace referencia al grupo de individuos que cuentan con la patología. Así mismo, la categoría "Controles" detalla el comportamiento de los individuos clínicamente definidos como sanos para la condición.

Con el fin de determinar la actividad enzimática de la $\beta$-glucosidasa en ausencia de la isoforma neutra, se usó el conduritol- $\beta$-epóxido (CBE $0,5 \mathrm{mM}$ ) en los blancos y se logró establecer que inhibe el 79,35 \% de la enzima pura. Lo anterior corrobora lo reportado en literatura sobre su importante acción inhibitoria para cuantificar la enzima de interés $(22,23,32)$, lo que nos permitió obtener un $0 \%$ de falsos negativos en el ensayo.

En cuanto a la comparación entre DBs y la prueba diagnóstica en leucocitos, no se logró obtener una correlación en términos estadísticos debido a la naturaleza de las muestras en cada protocolo. Como se mencionó previamente, la técnica de DBS incluye el cálculo de actividad enzimática sobre un volumen de sangre aproximado en el corte de papel de filtro, por lo que el resultado se expresa en $\mathrm{nmol} / \mathrm{ml} / \mathrm{h}$. Por otro lado, la prueba diagnóstica (gold standard) incluye el cálculo de actividad enzimática en una muestra concentrada de leucocitos que se corrige contra proteína total, por lo que el resultado se expresa en $\mathrm{nmol} / \mathrm{mg} / \mathrm{h}$ y lo convierte en una técnica mucho más confiable. No obstante, gráficamente se puede observar que sí existe una relación equiparable entre los dos métodos (Figura 2).

El análisis estadístico no mostró diferencias significativas entre las actividades enzimáticas de los pacientes de Colombia y Perú ( $\mathrm{p}>0,05)$. Sin embargo, a través del análisis de los blancos se pudo determinar que la isoforma neutra podría ser de aproximadamente el doble en los individuos peruanos en comparación con los pacientes colombianos, por lo que recalcamos la importancia de usar el CBE como inhibidor para cuantificar únicamente la BGLU que está relacionada con la enfermedad. 


\section{Conclusión}

Los resultados de este estudio ofrecen los valores de referencia para individuos sanos y afectados por la enfermedad de Gaucher en población colombiana. En este sentido, el tamizaje de alto riesgo que reportamos permite la identificación temprana de los pacientes potencialmente afectados por esta condición, de modo que los acerca a un diagnóstico temprano que se deriva en un tratamiento a tiempo, lo que mejora notablemente su calidad de vida y la de sus familias. A través de este método, se puede analizar un mayor número de muestras con un costo más favorable, puesto que se trata de una recolección en fase sólida que no necesita de requerimientos adicionales para su transporte $y$ almacenamiento. Además, se trata de un protocolo menos invasivo para el paciente y aseguramos el aprovechamiento total de la muestra a través del eluido que permite realizar varios ensayos de manera simultánea, lo que favorece en gran medida una orientación clínica más acertada. Por medio de este protocolo se puede conocer la real incidencia de la enfermedad en la población, debido a que acerca el laboratorio a las personas que viven en territorios de difícil acceso, por lo que podría aumentar el número de casos detectados. Más aún, dadas las características de la prueba, logramos beneficiar no solo a los pacientes colombianos, sino también a la población de países vecinos. Tal fue el caso de las muestras de Perú, a partir de las cuales concluimos que en dicha población existe una proporción cercana al doble de la isoforma neutra (no relacionada con la enfermedad de Gaucher, pero que sí degrada el sustrato artificial), de modo que resaltamos la importancia de usar el CBE para no incurrir en el error de cuantificar falsos negativos.

Reportamos una prueba de aproximación diagnóstica altamente eficaz con la cual se obtuvo un $0 \%$ de falsos negativos y aproximadamente un $10 \%$ de falsos positivos. En este apartado, es fundamental destacar la importancia de la leucopenia como un factor que afecta la actividad enzimática, razón por la cual el paso por seguir consta de cuantificar la $\beta$-glucosidasa teniendo en cuenta el número total de leucocitos para dichos pacientes, a fin de que se reduzca todavía más la tasa de falsos positivos.

Por último, cabe resaltar que el protocolo de DBs tiene el objetivo de facilitar la identificación temprana de los pacientes potencialmente afectados con la enfermedad, que paso seguido deben ser diagnosticados a través de la técnica de gold standard. Así mismo, todos los individuos que se encuentren con actividades enzimáticas en el punto de corte y posibles leucopénicos deben ser reconfirmados.

\section{Agradecimientos}

El presente estudio se llevó a cabo con la colaboración del Departamento de Ciencias Biológicas de la Universidad de los Andes.

\section{Referencias}

1. Palau F. Rare diseases, an emergent paradigm in the medicine of the XXI century. Med Clin (Barc) [Internet]. 2010;134(4):161-168. DoI: http://dx.doi.org/10.1016/j.medcli.2009.06.038

2. Pérez-Calvo JI, coordinador. Actualización en enfermedad de Gaucher [Internet]. Fundación Española de Enfermedades Lisosomales (FEEL) y Sistema Nacional de Salud, Comisión de Formación Continuada (CFC). 2008;70. Disponible en: http://2011.elmedicointeractivo.com/Documentos/doc/GAUCHER.pdf

3. Rua-Elorduy MJ. Enfermedades metabólicas lisosomales. Manifestaciones osteoarticulares. Protoc Diagnter Pediatr [Internet]. 2014;(1):231-239. Disponible en: https:/www.academia.edu/27694493/Enf_metabolicas_lisosomales

4. Uribe A, Giugliani R. Selective screening for lysosomal storage diseases with dried blood spots collected on filter paper in 4,700 high-risk Colombian subjects. JIMD Rep [Internet]. 2013;(11):107-116. DoI: https:// doi.org/10.1007/8904_2013_229

5. Wilches R, Vega H, Echeverri O, Barrera LA. Los haplotipos colombianos de la mutación N370S causante de la enfermedad de Gaucher pueden provenir de un haplotipo ancestral común. Biomédica [Internet]. 2006;26(3):434-441. Disponible en: https://bit.ly/3n4P$\mathrm{J} 5 \mathrm{r}$

6. Michelin K, Wajner A, Bock H, Fachel Â, Rosenberg R, Flores-Pires $\mathrm{R}$, et al. Biochemical properties of $\beta$-glucosidase in leukocytes from patients and obligated heterozygotes for Gaucher disease carriers. Clin Chim 
Acta [Internet]. 2005;362(1-2):101-9. DoI: https://doi. org/10.1016/j.cccn.2005.06.010

7. Lozano-Bernal JE. Enfermedad de Gaucher. Casuística del Tolima. Acta Médica Colomb [Internet]. 2006;31(4):416-421. Disponible en: http://www. scielo.org.co/scielo.php?script=sci_arttext\&pi$\mathrm{d}=$ S0120-24482006000400005

8. Sánchez KL, Quintana AN, Carreras IN, Otero AG, Svarch E, García SMH, et al. Aspectos clínicos, bioquímicos, moleculares y tratamiento de 2 pacientes con enfermedad de Gaucher. Rev Cuba Hematol Inmunol y Hemoter [Internet]. 2010;26(1):54-61. Disponible en: https://bit.ly/33e2126

9. Colquicocha-Murillo M, Cucho-Jurado J, Eyzaguirre-Zapata RM, Manassero-Morales G, Moreno-Larrea M del C, Salas-Arbizu KL, et al. Guía para diagnóstico y tratamiento de la enfermedad de Gaucher. Rev Médica Hered [Internet]. 2015;26(2):103-121. DoI: https://doi.org/10.20453/rmh.v26i2.2447

10. Grabowski GA, Horowitz M. 2 Gaucher's disease: molecular, genetic and enzymological aspects. Baillieres Clin Haematol [Internet]. 1997;10(4):635-656. DoI: https://doi.org/10.1016/S0950-3536(97)80032-7

11. Grabowski GA. Lysosomal storage disease 1. Phenotype, diagnosis, and treatment of Gaucher's disease. The Lancet [Internet]. 2008 [citado 2020 ene. 21];(372):1263-1271. DOI: https://doi.org/10.1016/ S0140-6736(08)61522-6

12. Liou B, Kazimierczuk A, Zhang M, Scott CR, Hegde RS, Grabowski GA. Analyses of variant acid-glucosidases effects of Gaucher disease mutations. J Biol Chem [Internet]. 2006 [citado 2020 ene. 21];281(7):4242-4253. DOI: https://doi.org/10.1074/jbc.M511110200

13. Grabowski GA. Gaucher disease: lessons from a decade of therapy. J Pediatr [Internet]. 2004;144(5 Suppl.):S15-S19. DOI: https://doi.org/10.1016/j. jpeds.2004.01.050

14. Stroppiano M, Calevo MG, Corsolini F, Cassanello M, Cassinerio E, Lanza F, et al. Validity of $\beta$-d-glucosidase activity measured in dried blood samples for detection of potential Gaucher disease patients. Clin Biochem [Internet]. 2014;47(13-14):1293-1296. DOI: http://dx.doi.org/10.1016/j.clinbiochem.2014.06.005

15. XIII Congreso Colombiano de Genética Humana y VII Congreso Internacional. Genética médica y dismorfología. Latin American Journal of Human Genetics [Internet]. 2014;2(1):44-136. Disponible en: https:// latinhumangenetics.com/pdfs_documents/LatinHuman_9resumenes.pdf
16. Sibille A, Eng CM, Kim SJ, Pastores G, Grabowski GA. Phenotype/genotype correlations in Gaucher disease type I: clinical and therapeutic implications. Am J Hum Genet [Internet]. 1993 [citado 2020 ene. 21];52(6):1094-1101. Disponible en: https://www.ncbi. nlm.nih.gov/pmc/articles/PMC1682271/

17. Bennett LL, Mohan D. Gaucher disease and its treatment options. Ann Pharmacother [Internet]. 2013;47(9):1182-1193. DoI: https://doi. org/10.1177/1060028013500469

18. Bennett LL, Turcotte K. Eliglustat tartrate for the treatment of adults with type 1 Gaucher disease. Drug Des Devel Ther [Internet]. 2015;9:4639-4647. DoI: https://doi.org/10.2147/DDDT.S77760

19. Wenger DA, Clark C, Sattler M, Wharton C. Synthe-

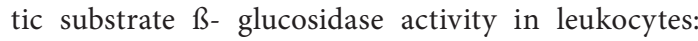
a reproducible method for the identification of patients and carriers of Gaucher's disease. Clin Genet [Internet]. 1978;13(2):145-153. DoI: https://doi. org/10.1111/j.1399-0004.1978.tb04242.x

20. Raghavan SS, Topol J, Kolodny EH. Leukocyte $\beta$-glucosidase in homozygotes and heterozygotes for Gaucher disease. Am J Hum Genet [Internet]. 1980;32(2):158173. Disponible en: https://www.ncbi.nlm.nih.gov/ pmc/articles/PMC1686022/

21. Daniels L, Glew R. B-glucosidase assays in the diagnosis of Gaucher's disease. Clin Chemestry [Internet]. 1982;28(4):569-577. DoI: https://doi.org/10.1093/clinchem/28.4.569

22. Uribe A, Arévalo I, Pacheco N. Over-expression of beta-glucosidase isoforms related to false negatives in diagnostic tests for Gaucher disease. Revista de Gastroenterología del Perú [Internet]. VIII Congreso de Errores Innatos del Metabolismo y Pesquisa Neonatal. Cusco, Perú. 2011;31(1):82-83. Disponible en: https:// bit.ly/2SayWzJ

23. Uribe A, Pacheco N. A modified method for the determination of acid beta-glucosidase in dried blood spot samples as a diagnostic approach in the andean countries: preliminary results. En: Proceedings of the 11th European Working Group on Gaucher Disease (EWGGD) Haifa, Israel [Internet]. 2014. DoI: https:// doi.org/10.13140/RG.2.2.24133.14567

24. Yıldırım Sözmen E, Dondurmacı M, Kalkan Uçar S, Çoker M. False positive diagnosis of lysosomal storage disease based on dried blood spot sample; leucocyte number of a challenging factor. J Pediatr Res [Internet]. 2018;5(Suppl. 1)17-21. DOI: https://doi. org/10.4274/jpr.33042 
25. Chamoles NA, Blanco M, Gaggioli D, Casentini C. Gaucher and Niemann-Pick diseases - enzymatic diagnosis in dried blood spots on filter paper: retrospective diagnoses in newborn-screening cards. Clin Chim Acta [Internet]. 2002;317(1-2):191-197. Dor: https://doi.org/10.1016/S0009-8981(01)00798-7

26. Civallero G, Michelin K, de Mari J, Viapiana M, Burin $\mathrm{M}$, Coelho JC, et al. Twelve different enzyme assays on dried-blood filter paper samples for detection of patients with selected inherited lysosomal storage diseases. Clin Chim Acta [Internet]. 2006;372(1-2):98-102. DoI: https://doi.org/10.1016/j.cca.2006.03.029

27. Peters SP, Coyle P, Glew RH. Differentiation of $\beta$-glucocerebrosidase from $\beta$-glucosidase in human tissues using sodium taurocholate. Arch Biochem Biophys [Internet]. 1976;175(2):569-582. DOI: https://doi. org/10.1016/0003-9861(76)90547-6

28. Rodrigues MDB, de Oliveira AC, Müller KB, Martins AM, D’Almeida V. Chitotriosidase determination in plasma and in dried blood spots: a comparison using two different substrates in a microplate assay.
Clin Chim Acta [Internet]. 2009;406(1-2):86-88. DOI: http://dx.doi.org/10.1016/j.cca.2009.05.022

29. Reuser AJ, Verheijen FW, Bali D, van Diggelen OP, Germain DP, Hwu WL, et al. The use of dried blood spot samples in the diagnosis of lysosomal storage disorders - Current status and perspectives. Mol Genet Metab [Internet]. 2011;104(1-2):144-148. DoI: https:// doi.org/10.1016/j.ymgme.2011.07.014

30. Daitx VV, Mezzalira J, Goldim MP de S, Coelho JC. Comparison between alpha-galactosidase A activity in blood samples collected on filter paper, leukocytes and plasma. Clin Biochem [Internet]. 2012;45(15):12331238. DoI: http://dx.doi.org/10.1016/j.clinbiochem.2012.04.030

31. Shapira E. Biochemical genetics: a laboratory manual. Oxford University Press; 1989. 145 p.

32. Olivova P, Cullen E, Titlow M, Kallwass H, Barranger J, Zhang K, et al. An improved high-throughput dried blood spot screening method for Gaucher disease. Clin Chim Acta [Internet]. 2008;398(1-2):163-164. DoI: http://dx.doi.org/10.1016/j.cca.2008.08.024 